\title{
Coincidencia de hallazgos endoscópicos e histológicos en el diagnóstico de esófago de Barrett
}

\author{
Coincidence of endoscopic and histological findings in the diagnosis of Barrett's esophagus
}

Alberto Piamo Morales', Isnerio Arzuaga Anderson', Lourdes Palma Machado', Digna Chávez Jiménez', Daisy Ferrer Marrero²

\section{Resumen}

Introducción: el esófago de Barrett es una alteración en la cual la mucosa esofágica se transforma desde un epitelio escamoso a un epitelio columnar con metaplasia intestinal. Endoscópicamente esta lesión se corresponde con la presencia de una mucosa columnar de color rojo salmón por encima de la unión gatroesofágica. Para su diagnóstico se requiere de la sospecha endoscópica y la confirmación histológica. Objetivo: determinar los niveles de coincidencia entre los diagnósticos endoscópicos y las características histopatológicas del esófago de Barrett, a partir del análisis de una serie de casos. Métodos: se realizó un estudio retrospectivo, en el Hospital Clínico Quirúrgico "Joaquín Albarrán" de La Habana, Cuba entre enero de 2017 a junio de 2019, obteniéndose los datos de los registros de biopsias e historias clínicas. Se realizaron cálculos de frecuencias absolutas y relativas y, para evaluar la fuerza de coincidencia entre endoscopia e histología, se utilizó el estadístico Chi cuadrado. Resultados: en los 67 casos con diagnóstico endoscópico la coincidencia con histopatológica fue del 44,8\%, en el restante 55,2\% de los pacientes las lesiones diagnosticadas fue esofagitis crónica ( $p=0,005$ ). Conclusiones: el esófago de Barrett es una de las entidades clínicas con una epidemiología de alta variabilidad y su diagnóstico endoscópicos requiere de la confirmación histológica ya que existe una baja coincidencia entre el diagnóstico endoscópico e histopatológico del EB el cual es el estándar de oro para el diagnóstico de EB.

Palabras clave: endoscopia; esófago de Barrett; reflujo gastroesofágico; displasia; metaplasia.

\begin{abstract}
Background: Barrett's oesophagus is an alteration in which the oesophagal mucosa is transformed from squamous epithelium to columnar epithelium with intestinal metaplasia. Endoscopically this lesion corresponds to the presence of a salmon-red columnar mucosa above the gastroesophageal junction. For its diagnosis, endoscopic suspicion and histological confirmation are required.

Objective: To determine the levels of coincidence between the endoscopic diagnoses and the histopathological characteristics of Barrett's oesophagus, from the analysis of a series of cases. Methods: A retrospective study was carried out at the "Joaquín Albarrán" Surgical Clinical Hospital in Havana, Cuba, between January 2017 and June 2019, obtaining data from biopsy records and medical records. Absolute and relative frequency calculations were performed and, to evaluate the force of coincidence between endoscopy and histology, the Chi-square statistic was used. Results: In the 67 cases with endoscopic diagnosis, the coincidence with histopathology was $44.8 \%$, in the remaining $55.2 \%$ of the patients the lesions diagnosed were chronic esophagitis $(p=0.005)$. Conclusions: Barrett's oesophagus is one of the clinical entities with the epidemiology of high variability, and its endoscopic diagnosis requires histological confirmation since there is a low coincidence between the endoscopic and histopathological diagnosis of EB which is the gold standard for EB diagnosis.
\end{abstract}

Keywords: Endoscopy; Barrett's oesophagus; gastroesophageal reflux; dysplasia; metaplasia.

Fecha de envío: 2020-05-09 - Fecha de aceptación: 2020-12-11

(1) Hospital Clínico Quirúrgico Docente "Joaquín Albarrán", La Habana-Cuba.

(2) Facultad de CM "ICBP Victoria de Girón". Universidad de Ciencias Médicas. La Habana. Cuba.

Correspondencia:b51amazonas@gmail.com 
Piamo et al.

\section{Introducción}

El esófago de Barrett (EB) es una condición adquirida que consiste en una lesión grave de la mucosa esofágica y cuya importancia radica en la posible evolución hacia el adenocarcinoma (González et al., 2012).

Si bien la esperanza de vida no se acorta directamente debido a la $E B$, ya que la mayoría de los pacientes fallecen por causas distintas al adenocarcinoma de esófago (ACE) (Sikkema et al., 2010), dicho diagnóstico conlleva importantes implicaciones económicas, de seguro médico y de gestión.

Aun cuando la confirmación del diagnóstico de EB es mediante el estudio anatomopatológico, se acepta que endoscópicamente se pueda sospechar esta entidad y en consecuencia realizar las tomas de las muestras adecuadas en número y extensión, lo cual incrementa la sensibilidad y especificidad para ofrecerle al paciente un óptimo seguimiento y curación. Bajo esta visión, el objetivo de este estudio ha sido, determinar los niveles de coincidencia entre los diagnósticos endoscópicos y las características histopatológicas del esófago de Barrett, a partir del análisis de una serie de casos.

\section{Materiales y métodos}

Se realizó un estudio retrospectivo, en el Hospital Clínico Quirúrgico "Joaquín Albarrán" de La Habana, Cuba para el cual se revisaron las solicitudes de biopsias en el período comprendido entre enero de 2017 a junio de 2019, para seleccionar aquellos casos en los cuales el especialista endoscopista estableció el diagnóstico de esófago de Barrett, o existía el diagnostico anatomopatológico de esófago de Barrett. Posteriormente se filtraron dichos casos en la base de datos del Servicio de Anatomía Patológica, obteniéndose los diagnósticos histopatológicos completos.

El diagnostico endoscópico se realizó siguiendo los criterios de la clasificación de Praga (Prateek, 2006), la cual establece dos aspectos: la extensión circunferencial $(C)$ y la extensión máxima en el eje longitudinal (M).

Criterios histológicos para el diagnóstico de esófago de Barrett fue la presencia de metaplasia intestinal que consiste en un epitelio columnar con células caliciformes bien formadas (Spechler et al., 2011). El grado de displasia se determinó evaluando la citología (características nucleares y citoplásmicas), la arquitectura (relación de las glándulas y la lámina propia) y el grado de maduración de la superficie (comparación del tamaño nuclear dentro de las criptas con el tamaño nuclear en la superficie de la mucosa). De acuerdo a estos elementos citológicos se establecieron dos tipos de lesión: displasia de bajo grado donde la característica más importante es la atipia que se extiende a la superficie de la mucosa y una maduración superficial mínima o ausente; y displasia de alto grado, donde los cambios citológicos son graves con núcleos marcadamente agrandados en la superficie, pleomorfismo pronunciado y al menos pérdida focal de la polaridad nuclear. Se pierde la maduración superficial. Incremento de las mitosis y mitosis atípicas (Booth \& Thompson, 2012).

Con la finalidad de completar los datos clínicos y endoscópicos se extrajeron del archivo las historias clínicas correspondientes. Se obtuvo un total de 74 pacientes, excluyendo a 4 casos en los que no se pudo completar los datos respectivos.

Una vez recopilados los datos, estos fueron almacenados en una base de datos, la cual facilitó el cálculo de frecuencias absolutas y relativas $y$, para evaluar la fuerza de coincidencia entre endoscopia e histología, se utilizó el estadístico Chi cuadrado.

\section{Resultados}

De los 74 pacientes estudiados, la proporción de hombres y mujeres fue de 1:1 (50\% para cada sexo). En un rango de edad de 18 a 80 años, el promedio fue de 55,8 $\pm 15,9$ años; siendo el grupo entre 62 a 72 años el de mayor predominio en un $28,4 \%$. Clínicamente, el $98 \%$ de los pacientes consultó por sín

Para el diagnóstico endoscópico se empleó la clasificación de Praga, mediante la cual se diagnosticaron un total de 67 casos, de ellos el $38,8 \%$ eran del tipo segmento corto y $61,2 \%$ de segmento largo, predominando el primero en el sexo femenino $(69,2 \%)$ y el segundo tipo en el sexo masculino $(65,9 \%)(p=0,005)$.

Tabla 1: Clasificación del esófago de Barrett según extensión de las lengüetas por sexo.

\begin{tabular}{l|c|c|c|c|c|c}
\hline \multirow{2}{*}{ SEGMENTO } & \multicolumn{4}{|c|}{ SEXO } & \multicolumn{2}{c}{ Total } \\
\cline { 2 - 6 } & \multicolumn{2}{|c|}{ Femenino } & \multicolumn{2}{c}{ Masculino } & \multicolumn{2}{c}{} \\
\cline { 2 - 7 } & $N^{\circ}$ & $\%$ & $N^{\circ}$ & $\%$ & $N^{\circ}$ & $\%$ \\
\hline Corto & 18 & 69,2 & 8 & 30,8 & 26 & $\mathbf{3 8 , 8}$ \\
\hline Largo & 14 & 34,1 & 27 & 65,9 & 41 & 61,2 \\
\hline Total & 32 & 47,8 & 35 & 52,2 & 67 & 100 \\
\hline
\end{tabular}

Histológicamente, se realizó el diagnóstico de EB en el 44,8\% (37) de los casos, en los cuales se pudo constatar la presencia de metaplasia intestinal completa, la cual estuvo definida por la presencia de un epitelio compuesto principalmente de células columnares y células caliciformes (Figura 1). 


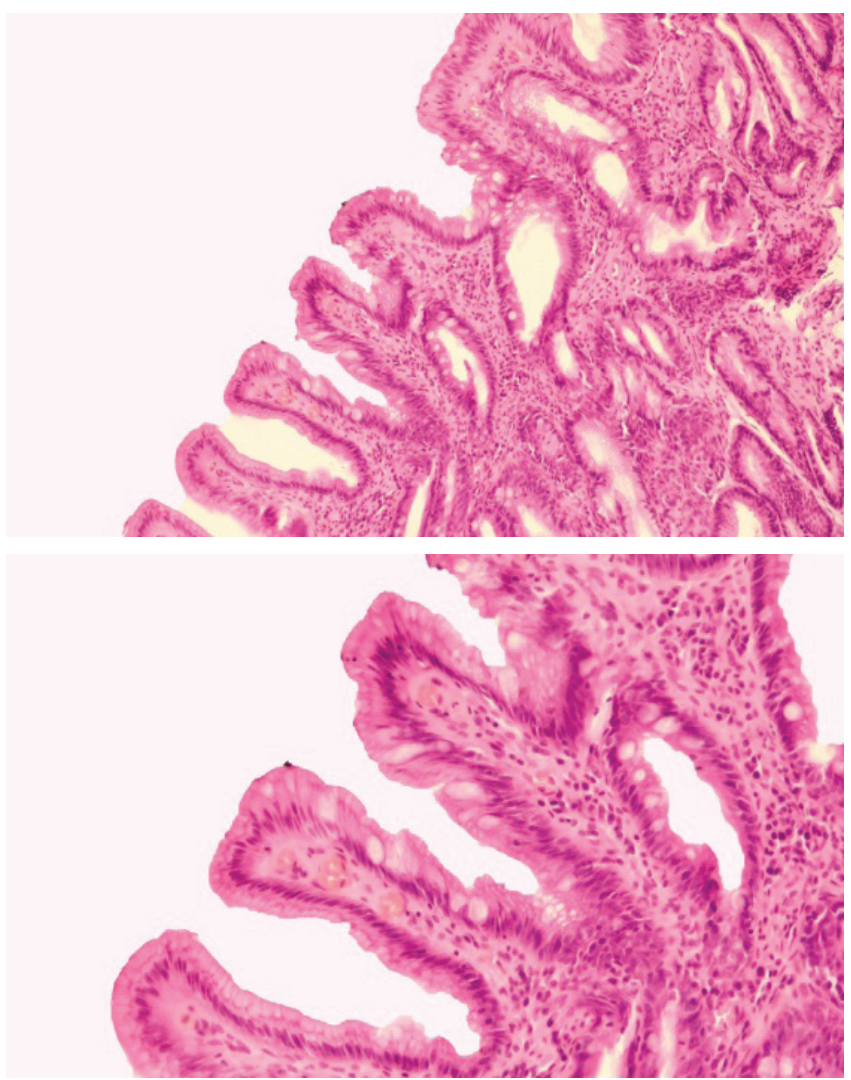

Figura 1: Mucosa esofágica con metaoplasia intestinal completa (10X), con presencia de células caliciformes (40X).

El sexo donde se presentó la mayor frecuencia de casos, fue el femenino $(56,8 \%)$ mientras que en hombres el EB se presentó sólo en el $43,2 \%$ de los casos. En cuanto al grupo de edad donde se observó la mayor frecuencia de casos fue el de 62 a 72 años $(35,1 \%)$ con un predominio del sexo femenino (Figura 2).

De los 37 casos con diagnóstico histológico, el 24,3\% presentó como hallazgos asociados, esofagitis crónica; el 2,7\%, acantosis epitelial y coilocitosis; y el 2,7\%, helicobacter pylori.

En cuanto a la presencia de displasia, esta solo se halló en el 5,41\% de los casos, siendo todas ellas ligeras (bajo grado).

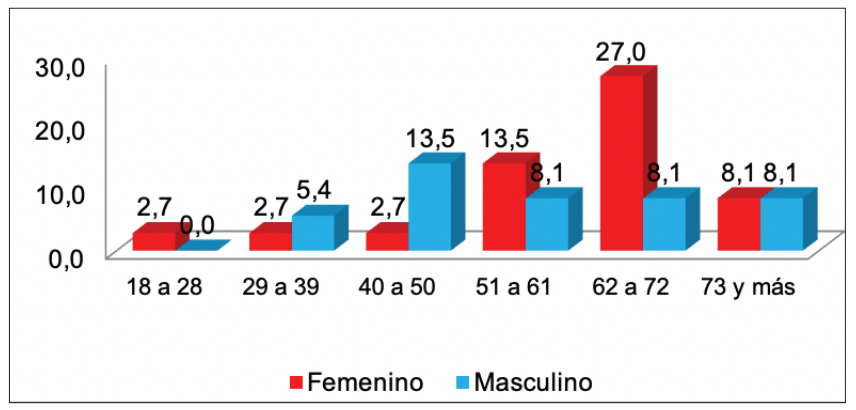

Figura 2: Distribución según grupos de edad y diagnóstico histológico de esófago de Barrett.
Ante la sospecha endoscópica de 67 casos con EB, hubo una coincidencia con el diagnóstico histopatológico de sólo el $44,8 \%$ de los casos (Tabla 2). En el restante $55,2 \%$ de los pacientes las lesiones diagnosticadas fueron: esofagitis crónica $(40,5 \%)$, con diferentes grados de intensidad del infiltrado inflamatorios incluyendo presencia de hiperplasia linfoide focal reactiva; procesos reactivos como acantosis, hiperqueratosis y lesiones hiperplásicas como el caso de un papiloma con coilocitosis ( $p=0,005)$.

En el estudio de la correlación endoscópica-histopatológica con respecto a la extensión del segmento, permitió observar que el $34,6 \%$ de los pacientes con un diagnóstico de EB de segmento corto tuvieron una correlación histopatológica, sin embargo, la mayoría de los pacientes (61\%) con EB de segmento largo presentaron una correlación.

Hubo 7 pacientes (18,9\%) en los cuales se realizó el diagnóstico histológico y no se había sospechado EB en el estudio endoscópico, de éstos, el 13,51\% (5) eran del tipo segmento corto y el 5,41\% (2) del tipo segmento largo.

Tabla 2: Tabla de contingencia.

\begin{tabular}{c|c|c|c|c|c|c}
\hline \multirow{2}{*}{ Diagnóstico Histológico } & \multicolumn{4}{|c}{ Diagnóstico endoscópico } & \multicolumn{2}{c}{ Total } \\
\cline { 2 - 7 } & $\mathrm{Si}$ & $\%$ & No & $\%$ & No & $\%$ \\
\hline $\mathrm{Si}$ & 30 & 44,8 & 7 & 100 & 37 & 50 \\
\hline No & 37 & 55,2 & 0 & 0,0 & 37 & 50 \\
\hline Total & $\mathbf{6 7}$ & $\mathbf{1 0 0}$ & $\mathbf{7}$ & $\mathbf{1 0 0}$ & $\mathbf{7 4}$ & $\mathbf{1 0 0}$ \\
\hline
\end{tabular}

\section{Discusión}

La frecuencia de EB descrita por Gonzalo-Marín et al. (2010) para la población general es de 0,9 a 4,5\% en función de la población de estudio y de los criterios de diagnóstico empleados. Un estudio alemán encontró que la prevalencia de EB es del $4,2 \%$, y otro estudio realizado en Japón informó una prevalencia del 0,3\% al 0,6\% (Sharma et al., 2000). En la presente investigación la incidencia de EB durante el periodo de 2017 a 2019 fue de 74 casos.

Con respecto al sexo, Anido et al. (2013) hallaron un predominio de EB en hombres del $61,3 \%$ y no hallaron diferencias estadísticamente significativas entre la edad y los diferentes tipos de Barrett ( $p=0,351) ; y$ en el estudio de Bahadır et al. (2015) de 11 pacientes con EB, la edad media fue de 46 años. Kasprzyk et al. (2016) hallaron un promedio de edad de pacientes con EB de 59,88 $\pm 12,09$ años.

En la presente investigación predominaron los pacientes entre 62 a 72 años, calculándose una edad media de 55,8 $\pm 15,9$ años, más baja que lo reportado Kasprzyk et al. 
Endoscópicamente el EB se divide de acuerdo a la extensión de la lesión en, segmento largo $(>3 \mathrm{~cm}$ ) y segmento corto $(<3 \mathrm{~cm})$, dicha distinción es necesaria mantenerla dado que la mayor parte de la información actualmente disponible sobre la epidemiología y el riesgo de transformación neoplásica del EB proviene de estudios en los que sólo se ha incluido a pacientes con segmentos largos (Pera \& Grande, 2002). Se reconoce una tercera variante, denominada segmento ultracorto, no visible endoscópicamente, pero con presencia de epitelio columnar confirmado histológicamente (Sharma et al., 1998), este segmento mide escasos milímetros y se encuentra situado en íntimo contacto con la UGE, la cual se observa mejor con la magnificación y el uso de cromoendoscopia, tanto electrónica como química, con posibles etiologías diferentes, como concluyen los estudios de Matsuzaki et al. (2011).

Pohl et al. (2016) halló en el estudio de 1017 pacientes con ACE recién diagnosticado, 573 (56\%) EB de segmento largo, 240 (24\%) y 204 (20\%) segmento ultracorto, así mismo, reporto que las tasas anuales de transición de cáncer para pacientes con esófago de Barrett largo, corto y ultra corto fueron de $0,22 \%, 0,03 \%$ y $0,01 \%$, respectivamente.

En un estudio realizado por Padda \& Ramirez (2001) las tasas de confirmación histopatológica de EB de segmento corto y largo fueron del 38 y $75 \%$, respectivamente. Al igual que en estos, en el presente estudio, solo el $34,6 \%$ de los pacientes con un diagnóstico de EB de segmento corto durante la endoscopia tenían un EB confirmado histopatológicamente, mientras que la mayoría de los pacientes categorizados como EB de segmento largo durante la endoscopia tenían un EB histológico.

En esta investigación los casos de EB de segmento largo fueron de $61,2 \%$ de los casos con predominio en el sexo masculino y el $38,8 \%$ fueron de segmento corto con predominio femenino (Tabla 1).

Es posible que la buena concordancia endoscópica-histológica en EB de segmento largo se deba a la extensión de las lesiones, las cuales pueden ser más evidentes para el endoscopista.

El reconocimiento preciso de la displasia en el EB es crucial debido a su valor pronóstico fundamentalmente (Yin et al., 2019). En esta serie de casos la presencia de displasia solo se encontró en 5,41\% de los casos; lo cual se corresponde con lo hallado por Meining et al. (2004) quienes reportaron que de 235 pacientes positivos para el EB la displasia fue muy baja.

En relación a la presencia y grado de displasia, Kasprzyk et al. (2016) observaron que $16,47 \%$ de los casos estudiados tenían DBG, sin ningún caso con displasia de alto grado (DAG); y en la investigación de Angarita et al. (2008) predominó el esófago de Barrett sin displasia en $37,07 \%$. En el presente estudio la displasia se constató solo en el 5,41\% de los casos, siendo todas ellas ligeras (bajo grado).
Los médicos deben comprender la importancia del posible nivel de incertidumbre involucrado en el diagnóstico de displasia en muestras de biopsia de mucosa obtenidas del esófago o la UGE. Desafortunadamente, en la comunidad de patología general, existe una tendencia a sobre-diagnosticar la displasia, particularmente en el caso de la DAG. Por ejemplo, en un estudio de 485 pacientes, casi un $40 \%$ de los pacientes que inicialmente fueron diagnosticados con DAG por un patólogo general se redujeron (11\% sin displasia, $12 \%$ de indefinida para la displasia, 16\% de DBG) cuando las muestras fueron revisadas por tres patólogos gastrointestinales experimentados (Kerkhof et al., 2007), es por ello que la recomendación es firme en la confirmación del diagnóstico histológico por un segundo evaluador (Ferro \& Martorell, 2018).

En cuanto a la detección de displasia, algunos estudios han sugerido que el uso de imágenes de banda estrecha y cromoendoscopia de aumento o biopsias dirigidas con azul de metileno facilita su detección; sin embargo, estas sugerencias son controvertidas (Anagnostopoulos et al., 2007).

En los últimos años se han producido un conjunto de avances en los métodos mínimamente invasivos que han mostrado buenos resultados en la detección de EB y podrían ser útiles para el cribado masivo con menores costos. Un ejemplo de esto es la citoesponja. Esta técnica ha demostrado ser segura y eficaz en el diagnóstico de EB. En una gran cohorte de pacientes, este método mostró una tasa de éxito del 93,9\% durante la deglución de la cápsula. La sensibilidad para el diagnóstico de EB se ha reportado entre 79,9\% y del $87,2 \%$ en pacientes con EB de segmento largo (> $3 \mathrm{~cm})$, con una especificidad del 92,4\% (Ross et al., 2015).

Otras técnicas menos invasivas es la endoscopia transnasal y la cápsula endoscópica esofágica. La endoscopia transnasal realizada de forma ambulatoria ha demostrado ser segura, con una eficacia comparable para la evaluación de la mucosa esofágica y tiempos de procedimiento y recuperación más cortos en comparación con la endoscopia convencional (Sami et al., 2015). La cápsula endoscópica esofágica ha demostrado ser segura, aunque la sensibilidad y especificidad para el diagnóstico de EB aún son bajas (Bhardwaj et al., 2009).

Los endoscopios de alta resolución con sistemas de alta definición han reemplazado estos dispositivos de resolución estándar mejorando la visualización de las anomalías de la mucosa (Kwon et al., 2009). Su uso en combinación con otras técnicas como la cromoendoscopia mejora la detección de metaplasia intestinal y DAG en pacientes con metaplasia columnar detectada (Sharma et al., 2003). 
La endoscopia de luz blanca de alta definición (HD-WLE) se ha utilizado en combinación con técnicas de imagen novedosas para la detección temprana de cambios en la mucosa que sugieren displasia. Aunque la combinación de estas técnicas puede no aumentar significativamente la detección de EB o displasia en comparación con HD-WLE sola, se pueden lograr biopsias dirigidas y se puede reducir el número de biopsias (Sharma et al., 2013).

La endomicroscopía láser confocal, permite la evaluación muy detallada del epitelio de la superficie, así como el patrón vascular de secciones seriadas de muestras gruesas in vivo. Se ha demostrado una sensibilidad y especificidad del $98,1 \%$ y $94,1 \%$, respectivamente (Singh et al., 2015).

La endomicroscopía láser volumétrica, este método utiliza luz infrarroja para producir imágenes transversales de alta resolución en tiempo real del esófago. Con este sistema se puede escanear una longitud de $6 \mathrm{~cm}$ del esófago en aproximadamente 90 segundos, proporcionando imágenes transversales de campo amplio de la superficie y del subsuelo con una resolución axial de $7 \mu \mathrm{m}$ y una profundidad de $3 \mathrm{~mm}$ (Trindade et al., 2016).

El conocimiento del sitio exacto de la biopsia y la impresión endoscópica son esenciales al evaluar las biopsias esofágicas. Los médicos endoscopistas son responsables de transmitir información sobre el sitio anatómico de las muestras de biopsia, pero en algunos casos puede ser difícil determinar el sitio preciso de la misma. En aproximadamente el $30 \%$ de las muestras de biopsia tomadas de la región de la UGE, los patólogos pueden determinar con certeza si se obtuvo una muestra del esófago distal y, por tanto, si los hallazgos representan la presencia de un epitelio columnar metaplásico en el esófago (Srivastava et al., 2007). Sin embargo, en el resto de los casos, los patólogos no pueden distinguir con certeza si el origen de la muestra de la biopsia fue esofágico o gástrico. En tales casos, la opinión de los endoscopistas es la fuente de información más confiable y, por lo tanto, la comunicación de esta información al patólogo es esencial.

Suele existir una discordancia entre el diagnóstico clínico-endoscopico e histopaotógico de EB (Takubo et al., 2009). Al respecto, Angarita et al. (2008) reportaron una coincidencia en el diagnóstico de $57,74 \%$ al analizar 433 biopsias.

Es posible que la correlación entre endoscopía y diagnóstico histológico obtenida en este estudio esté determinada por el hecho de que el número y ubicación de donde se obtienen las muestras no se corresponden con las normas internacionales.

Existen controversias con respecto a la correlación de la esofagitis y la EB. Bahadır et al. (2015) no encontraron diferencias estadísticamente significativas en las tasas de esofagitis de los pacientes con EB. Con la finalidad de hacer diagnósticos histológicos más precisos existen un conjunto de biomarcadores ya que, como lo describieron Hahn et al. (2009) el epitelio columnar esofágico metaplásico es inmunopositivo para los marcadores de diferenciación intestinal, DAS-1, villina y CDX2 en 30\%, 70\% y 43\% de los casos, respectivamente, lo que indica una evidencia de diferenciación intestinal a pesar de la ausencia de células de cubilete por morfológica evaluación.

Investigadores holandeses han evaluado prospectivamente el análisis inmunohistoquímico de p53, AMACR, ciclina A y SOX2 en pacientes con EB para caracterizar el valor predictivo de estos marcadores para la progresión neoplásica (Van Olphen et al., 2015). En un gran estudio prospectivo multicéntrico, se siguió a 625 pacientes con EB en un programa de vigilancia endoscópica durante una mediana de 6,7 años. Cincuenta pacientes (8\%) desarrollaron progresión neoplásica durante la vigilancia después de una mediana de seguimiento de 3,2 años. Se observó inmunopositividad a ciclina $\mathrm{A}$ en el $10 \%$ de las biopsias no displásicas, en el 33\% de las biopsias de DBG y en el 69\% de las biopsias de DAG y / o adenocarcinoma de esófago, y se asoció con un mayor riesgo de progresión neoplásica. Los mismos autores evaluaron la expresión de p53 y SOX2 en estudios anteriores, y estos marcadores demostraron un valor pronóstico similar (Van Olphen et al., 2016). En otro estudio prospectivo transversal de 175 pacientes con EB, la aneuploidía y la inmunopositividad de p53 o ciclina A tenían las asociaciones más fuertes con displasia (Di Pietro et al., 2015).

La hibridación in situ fluorescente (FISH) utiliza muestras de cepillado esofágico para predecir la progresión de EB y la respuesta al tratamiento. Este método utiliza sondas de ADN marcadas con fluorescencia para detectar la ganancia o pérdida de regiones cromosómicas específicas. Presenta una sensibilidad del $80 \%$ y una especificidad del $88 \%$ para identificar DAG y / o adenocarcinoma de esófago (Poneros et al., 2017).

Se ha desarrollado un ensayo comercial para uso con muestras de biopsia histológica. Tissue Cypher (Cernostics, Inc, Pittsburgh, Pensilvania, EE.UU.) el cual se está utilizando actualmente en la práctica clínica para proporcionar datos adicionales en pacientes con EB sobre su riesgo de progresión a cáncer de esófago. Este ensayo utiliza algoritmos para interpretar biomarcadores cuantitativos (inmunohistoquímica y FISH), datos de características de morfología celular y subcelular. El software extrae un total de 1184 características y / o muestras de biopsia de los biomarcadores y la morfología y luego se resumen como medidas múltiples. Con base en estos datos, se genera una puntuación de riesgo a 5 años para la progresión a DAG y / o adenocarcinoma de esófago (Trindade et al., 2019). 
Aun cuando se cuenta con los marcadores antes mencionados, en la actualidad, la histopatología sigue siendo el estándar de oro, ya que la alteración que determina las manifestaciones clínicas y el riesgo de desarrollar lesiones malignas solo pueden se precisada por medio del estudio microscópico. De tal manera que el reciente consenso de la Sociedad de Patología Gastrointestinal (Srivastava et al., 2017) no recomienda los biomarcadores para realizar el diagnóstico de EB.

A partir de las herramientas diagnosticas hasta aquí descritas, se puede desarrollar una vigilancia eficaz que permita el manejo adecuado del EB según el grado de displasia. Sin embargo, el futuro de la vigilancia en EB está migrando gradualmente hacia el riesgo, estratificando a las personas con mayor riesgo de progresión del cáncer en función de los factores de riesgo y los biomarcadores para priorizar a los pacientes con mayor riesgo de cáncer.

\section{Conclusiones}

El esófago de Barrett es una de las entidades clínicas con una epidemiología de alta variabilidad y su diagnóstico endoscópicos requiere de la confirmación histológica ya que existe una baja coincidencia entre el diagnóstico endoscópico e histopatológico del EB el cual es el estándar de oro para el diagnóstico de EB.

\section{Limitaciones del estudio}

Dado que la muestra fue seleccionada de forma intencional a partir de los casos con diagnostico endoscópico y/o histológico de EB, no se incluyeron casos sanos, lo cual impide el cálculo de la sensibilidad y especificidad.

\section{Referencias}

Anagnostopoulos GK, Yao K, Kaye P, Hawkey CJ. \& Ragunath K. (2007). Novel endoscopic observation in Barrett's esophagus using high resolution magnification endoscopy and narrow band imaging. Aliment Pharmacol Ther 26, 501-7.

Angarita O, Granados C. \& Ricaurte O. (2008). Frecuencia de esófago de Barrett en una serie de biopsias endoscópicas de esófago (2002-2007). Rev Col Gastroenterol 23.

Anido V, Cathcart F, Brizuelas R, García E, Díaz Z. \& Morera M. (2013). Factores de motilidad en el Esófago de Barrett, según diferente presentación endoscópica. Rev Haban Cienc Méd.12.

Bahadır E, Tolga D, Baris Y, Zeynep B. \& Hakan B. (2015). Utility of Endoscopy for Diagnosis of Barrett in a Non-Western Society: Endoscopic and Histopathologic Correlation. Int Surg. 100, 720-725.
Booth CL \&Thompson KS. (2012). Barrett's esophagus: A review of diagnostic criteria, clinical surveillance practices and new developments. Journal of gastrointestinal oncology. 3, 232-242.

Bhardwaj A, Hollenbeak CS, Pooran N \& Mathew A. (2009). A meta-analysis of the diagnostic accuracy of esophageal capsule endoscopy for Barrett's esophagus in patients with gastroesophageal reflux disease. The American journal of gastroenterology 104, 1533-1539.

Committee. (2009). High-resolution and high-magnification endoscopes. Gastrointestinal endoscopy 69, 399-407.

Di Pietro M, Boerwinkel DF, Shariff MK, Liu X, Telakis E, Lao-Sirieix P, Walker E, Couch G, Mills L, Nuckcheddy-Grant T, Slininger S, O'Donovan M, Visser, M, Meijer SL, Kaye PV, Wernisch L, Ragunath K, Bergman JJ \& Fitzgerald RC. (2015). The combination of autofluorescence endoscopy and molecular biomarkers is a novel diagnostic tool for dysplasia in Barrett's oesophagus. Gut 64, 49-56.

Ferro DI \& Martorell S. (2018). Enfermedad de Barrett. En F. Galindo y colab. Enciclopedia Cirugía Digestiva. 172, 1-17.

González N, Parra-Blanco A \& Cohen H. (2012). Esófago de Barrett: Actualización del tema. Acta Gastroenterológica Latinoamericana 42, 333-347.

Gonzalo-Marín J, Navarro-Jarabo J \& Pérez-Aisa A. (2010). Actualización en el manejo del Esófago de Barrett. RAPD. 33, 144-9.

Hahn HP, Blount PL, Ayub K, Das KM, Souza R \& Spechler S. (2009). Intestinal differentiation in metaplastic, non-goblet columnar epithelium in the esophagus. Am J Surg Pathol. 33, 1006-15.

Kasprzyk J, Cantero G, Díaz C \& Ferreira M. (2016). Concordancia de hallazgos endoscópicos e histológicos en pacientes con esófago de Barrett. Rev Nac. 8, 17-23.

Kerkhof M, van Dekken H, Steyerberg EW, Meijer GA, Mulder AH \& de Bruïne A. (2007). Grading of dysplasia in Barrett's esophagus: substantial interobserver variation between general and gastrointestinal pathologists. Histopathology 50, 920-7.

Kwon RS, Adler DG, Chand B, Conway JD, Diehl DL, Kantsevoy SV, Mamula P, Rodriguez SA, Shah RJ, Wong Kee Song LM \& Tierney WM, ASGE Technology Sharma P, Weston AP, Topalovski M, Cherian R, Bhattacharyya A \& Sampliner RE (2003). Magnification chromoendoscopy for the detection of intestinal metaplasia and dysplasia in Barrett's oesophagus. Gut 52, 24-27. 
Matsuzaki J, Suzuki H, Asakura K, Saito Y, Hirata K \& Takebayashi T. (2011). Etiological difference between ultrashort and short segment Barrett's esophagus. J Gastroenterol. 46, 332-8.

Meijer SL, Kaye PV, Wernisch L, Ragunath K, Bergman JJ \& Fitzgerald RC. (2015). The combination of autofluorescence endoscopy and molecular biomarkers is a novel diagnostic tool for dysplasia in Barrett's oesophagus. Gut, 64, 49-56.

Meining A, Ott R, Becker I, Hahn S, Mühlen J. \&Werner M. (2004). The Munich Barrett follow up study: suspicion of Barrett's esophagus based on either endoscopy or histology only — what is the clinical significance? Gut. 53, 1402-1407.

Padda S \& Ramirez F. (2001). Accuracy in the diagnosis of SSBE: the role of endoscopic experience. Gastrointest Endosc. 54, 605-608.

Pera M \& Grande L. (2002). Cáncer en el esófago de Barrett. GH Continuada. 1, 247-251.

Poneros JM, Faye AS, Barr Fritcher EG, Sen A, Anandasabapathy S, Bresalier RS, Marcon N, Turgeon DK, Appelman H, Normolle D, Morrison LE, Brenner DE \& Halling KC. (2017). A Multicenter Study of a Fluorescence In Situ Hybridization Probe Set for Diagnosing High-Grade Dysplasia and Adenocarcinoma in Barrett's Esophagus. Digestive diseases and sciences 62, 1216-1222.

Pohl H, Pech O, Arash H, Stolte M, Manner H \& May A. (2016). Length of Barrett's oesophagus and cancer risk: implications from a large sample of patients with early oesophageal adenocarcinoma. Gut. 65, 196-201.

Ross-Innes CS, Debiram-Beecham I, O'Donovan M, Walker E, Varghese $S$, Lao-Sirieix P, Lovat L, Griffin M, Ragunath $K$, Haidry R, Sami SS, Kaye P, Novelli M, Disep B, Ostler R, Aigret B, North BV, Bhandari P, Haycock A, Morris D, Attwood S, Dhar A, Rees C, Rutter MD, Sasieni PD \& Fitzgerald RC. Grupo de estudio BEST2. (2015) Evaluation of a minimally invasive cell sampling device coupled with assessment of trefoil factor 3 expression for diagnosing Barrett's esophagus: a multi-center case-control study. PLoS Med. 12, e1001780.

Sami SS, Dunagan KT, Johnson, ML, Schleck, CD, Shah, ND, Zinsmeister, AR, Wongkeesong, LM, Wang, KK, Katzka, DA, Ragunath, K, \& lyer, PG. (2015). A randomized comparative effectiveness trial of novel endoscopic techniques and approaches for Barrett's esophagus screening in the community. The American journal of gastroenterology 110, 148-158.

Sharma P, McCallum RW \& Lundell L. (2000). The geoprevalence of Barrett's esophagus. Presented at: Sixth OESO World Congress. Paris, France.
Sharma P, Morales TG \& Sampliner RE. (1998). Short segment Barrett's esophagus: the need for standardization of the definition and of endoscopic criteria. Am J Gastroenterol. 93, 1033-1036

Sharma P, Wani S, Rastogi A, Bansal A, Higbee A, Mathur S, Esquivel R, Camargo L \& Sampliner RE (2008). The diagnostic accuracy of esophageal capsule endoscopy in patients with gastroesophageal reflux disease and Barrett's esophagus: a blinded, prospective study. The American journal of gastroenterology $103,525-532$.

Sharma P, Hawes RH, Bansal A, Gupta N, Curvers W, Rastogi A, Singh M, Hall M, Mathur SC, Wani SB, Hoffman B, Gaddam S, Fockens P \& Bergman JJ (2013). Standard endoscopy with random biopsies versus narrow band imaging targeted biopsies in Barrett's oesophagus: a prospective, international, randomised controlled trial. Gut 62, 15-21.

Spechler SJ, Sharma P, Souza RF, Inadomi JM \& Shaheen NJ. (2011) American Gastroenterological Association medical position statement on the management of Barrett's esophagus. Gastroenterology. 140, 1084-91.

Sikkema M, de Jonge PJ, Steyerberg EW \& Kuipers EJ. (2010). Risk of esophageal adenocarcinoma and mortality in patients with Barrett's esophagus: a systematic review and meta-analysis. Clin Gastroenterol Hepatol. 8, 235-244.

Singh A \& Chak A. (2015). Advances in the management of Barrett's esophagus and early esophageal adenocarcinoma. Gastroenterology report, 3, 303-315.

Srivastava A, Odze RD, Lauwers GY, Redston M, Antonioli DA \& Glickman JN. (2007). Morphologic features are useful in distinguishing Barrett esophagus from carditis with intestinal metaplasia. Am J Surg Pathol. 31, 1733-1741.

Srivastava A, Appelman H, Goldsmith JD, Davison JM, Hart J \& Krasinskas AM. (2017). The Use of Ancillary Stains in the Diagnosis of Barrett Esophagus and Barrett Esophagus-associated Dysplasia: Recommendations from the Rodger C. Haggitt Gastrointestinal Pathology Society. Am. J. Surg. Pathol. 41, 8-21.

Takubo K, Vieth M, Aida J, Sawabe M, Kumagai Y \& Hoshihara Y. (2009). Differences in the Definitions Used for Esophageal and Gastric Diseases in Different Countries. Digestion. 80, 248-257.

Tan WK, di Pietro M \& Fitzgerald RC. (2017). Past, present and future of Barrett's oesophagus. EJSO. 43, 1148-1160. 
Piamo et al.

Trindade AJ, Smith MS \& Pleskow DK. (2016). The new kid on the block for advanced imaging in Barrett's esophagus: a review of volumetric laser endomicroscopy. Therapeutic advances in gastroenterology, 9, 408-416.

Trindade AJ, Navaneethan U, Aslanian HR, Bhutani MS, Krishnan K, Lichtenstein DR, Melson J, Pannala R, Parsi MA, Schulman AR, Sethi A, Trikudanathan G, Watson RR, Maple JT \& ASGE Technology Committee, (2019). Advances in the diagnosis and surveillance of Barrett's esophagus (with videos). Gastrointestinal endoscopy 90, 325-334.

Van Olphen S, Biermann K, Spaander MC, Kastelein F, Steyerberg EW, Stoop HA, Bruno MJ \& Looijenga LH. (2015). SOX2 as a novel marker to predict neoplastic progression in Barrett's esophagus. Am J Gastroenterol. 110, 1420-1428.
Van Olphen SH, Ten Kate FJ, Doukas M, Kastelein F, Steyerberg EW, Stoop HA, Spaander MC, Looijenga LH, Bruno MJ, Biermann K \& ProBar-Study Group. (2016). Value of cyclin A immunohistochemistry for cancer risk stratification in Barrett esophagus surveillance: $A$ multicenter case-control study. Medicine 95, 5402.

Yin F, Hernández D, Lai J \& Liu X. (2019). Histopathology of Barrett's Esophagus and Early-Stage Esophageal Adenocarcinoma: An Updated Review. Gastrointest. Disord. 1, 147-163. 